\title{
Focused abdominal compression as a means to control post-partum hysterectomy hemorrhage: A case report
}

\author{
Mayuri Ahuja ${ }^{1, *}$, S Gulati ${ }^{2}$, Neerja Goel ${ }^{3}$, Shehla Jamal ${ }^{4}$ \\ ${ }^{1}$ Senior Resident, ${ }^{2,3}$ Professor, ${ }^{4}$ Assistant Professor, Dept. of Obstetrics and Gynecology, School of Medical Sciences and \\ Research, Sharda University, Greater Noida, Uttar Pradesh, India
}

*Corresponding Author:

Email: soniaahuji@rediffmail.com

\begin{abstract}
Placenta accreta is a life threatening obstetric condition that requires multidisciplinary approach for its management. Presently we document one such case of placenta accreta diagnosed intraoperatively during caesarean section where patient continued to bleed despite peripartum hysterectomy and eventually hemostasis was achieved by focused vault compression.
\end{abstract}

Keywords: MRI, Posterior placenta accreta, Ultrasonography, Peripartum hysterectomy, Umbrella packing, Abdominal packing.

\section{Introduction}

Placenta accreta is a condition when part of placenta or entire placenta invades and is inseparable from the uterine wall or uterine scar. ${ }^{1}$ In placenta increta chorionic villi invade only the myometrium and Placenta accreta is probably the most common cause of cesarean hysterectomy. Other problems that could be associated are massive obstetric hemorrhage, coagulopathy, surgical injury to ureter, bladder, bowel, need for massive blood transfusion, acute transfusion reaction, electrolyte imbalance and renal failure. Diagnosis is generally by grey scale ultrasonography. ${ }^{2}$ Cesarean hysterectomy with placenta left in situ is the recommended management. ${ }^{3}$ Internal artery ligation, arterial embolization and abdominal packing are adjuncts to attain hemostasis. This case will highlight the effectiveness of focused abdominal compression in controlling post hysterectomy hemorrhage.in percreta villi invade the serosa of surrounding visceral structures.

\section{Case Report}

A 26-year-old third gravida with previous two deliveries by cesarean section presented at 40 weeks by (12 weeks scan) for further management. Patient conceived during lactational amenorrhea hence last menstrual period was not known. Obstetric history revealed L1: Male, Lower segment caesarean section done in view of breech, 7 years back. L2: female, LSCS done in view of previous LSCS with scar tenderness, 1 year back. On examination mild pallor was present, Pulse-80/min, Blood Pressure-100/70 mm $\mathrm{Hg}$, cardiovascular and respiratory system normal. On abdominal examination pfannenstiel incision scar present, uterus 34 weeks, relaxed with breech presentation, no scar tenderness elicited, liquor appeared to be reduced clinically. She was not in labor. Her ultrasonography showed single live fetus of 34 weeks, asymmetrical fetal growth restriction, anhydraminos with posterior placenta and estimated birth weight of $2 \mathrm{~kg}$. Color doppler study was normal. Patient was advised emergency caesarean section. Lab parameters: hemoglobin $=8.8 \mathrm{mg} / \mathrm{dl}$ and viral markers for HIV, Hepatitis B, anti HCV were negative. Patient and attendant explained the need of blood transfusion, ICU and NICU admission, the possible risks of bowel and bladder injury due to dense adhesions which could be encountered. Spinal anesthesia was given and abdomen was opened by pfannenstiel incision. Dense adhesions between rectus sheath and muscle were separated by sharp dissection. On the anterior lower segment of uterus fatty layer was present which opened with fingers. No liquor was present. Female baby was delivered as breech weighing $2 \mathrm{~kg}$. Placenta did not detach spontaneously and was found to be adherent to the posterior wall of uterus. Uterus was atonic and there was massive on going hemorrhage. Her blood pressure dropped to $90 / 60 \mathrm{~mm} \mathrm{Hg}$ and pulse $150 / \mathrm{min}$. Nor-adrenaline drip was started. Spinal anesthesia was converted to general anesthesia. Bilateral internal artery ligation was done followed by subtotal hysterectomy. Four units packed red blood cell (PRBC) and 4 units of thawed fresh frozen plasma (FFP) were transfused intraoperatively. The bleeding from the cervical stump continued despite using hemostatic agents and sutures. Due to non- availability of umbrella pack, four sponge holders and 2 mops used to compress the stump. The sponge holders were covered with sterile gauze and brought out from the incision site and skin was closed with interrupted sutures. Patient shifted to surgical ICU on ventilatory support. Four units (PRBC) and 2 units (FFP) given post operatively also on post-operative day 1: Patient on ventilatory support and nor- adrenaline drip. Patient developed pulmonary edema. Her prothrombin time $(\mathrm{PT})=23$ seconds, INR $=1.83$. Patient given 4 units of additional FFP for correction of coagulopathy. Fluid restriction and diuretics were given for correction of pulmonary edema. On post-operative day2: Patient still on ventilatory support and non - adrenaline drip. Chest was clear, vitals stable, urine output adequate, 
hemoglobin was $9 \mathrm{~g} / \mathrm{dl}, \mathrm{PT}=15$, INR= 1.3 Decision for removal of sponge holder and pack was taken after vigorous discussion with the anesthesiologist. On second laparotomy hemostasis was achieved after removal of the sponge holders. No evidence of necrosis was seen. No additional hemostatic sutures required. Intra-peritoneal drain was put and abdomen closed. On third post-operative day ventilator was weaned off and non-adrenaline drip tapered and then stopped. Intraperitoneal drain was removed on day 4. Her postoperative recovery was good and patient discharged on day 10.

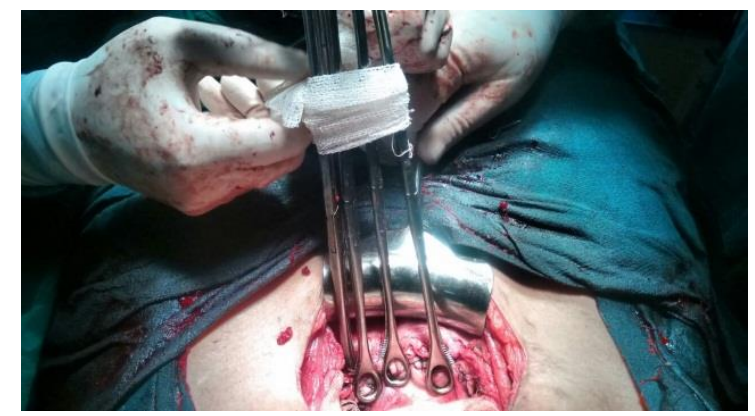

Fig. 1: Vaginal vault compression by sponge holder

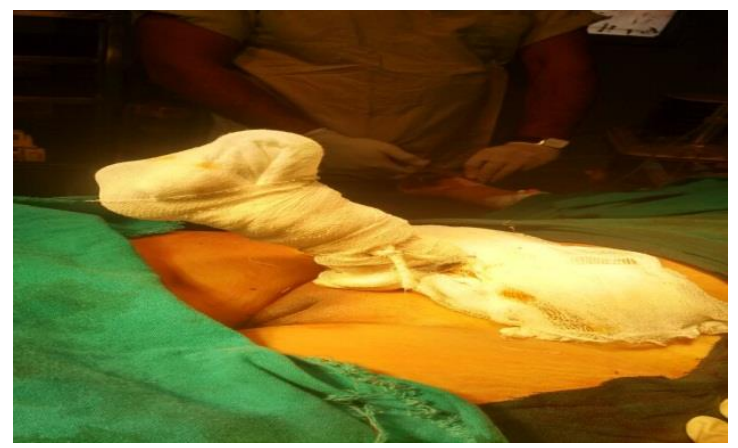

Fig. 2: Sponge holder covered with sterile gauze brought out from the incision site

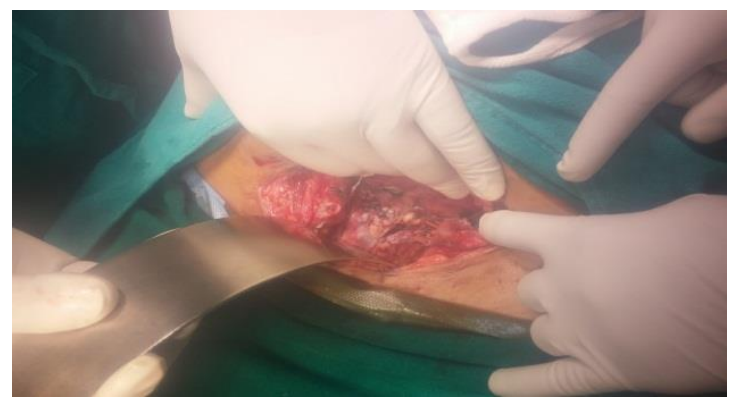

Fig. 3: Healthy vaginal vault after removal of the pack and the sponge holder

\section{Discussion}

The incidence of placenta accreta after first, second, third, fourth and fifth caesarean is $3 \%, 11 \%$, $40 \%, 61 \%$ and $67 \%$ respectively. ${ }^{4}$ Ultrasonography has sensitivity of $77-87 \%$ and specificity of $96 \%$-98\%in their diagnosis. ${ }^{5,6}$ The ultra-sonographic features suggestive of placenta accreta are irregular shaped lacunae within the placenta, thinning of myometrium overlying the placenta, loss of retroplacental space between placenta and bladder, increased vascularity of the uterine serosa bladder interface, turbulent blood flow through lacunae. ${ }^{5,6}$ Color doppler study does not add further to the diagnosis of placenta accrete. MRI can be used where diagnosis is not conclusive. Caesarean hysterectomy is the recommended management. Other measures to attain hemostasis include internal iliac artery ligation, arterial embolization and abdominal packing. Abdominal packing as a method of hemostasis for post hysterectomy hemorrhage has been used previously used to control haemorrhage. ${ }^{8-10}$ Insufficient pressure during packing can result in persistent ongoing bleeding and prolonged and tight pressure can result in abdominal compartment syndrome which would result in end organ damage. ${ }^{11}$ The biggest dilemma is what is the right time to remove these packs. Literature is scanty on this issue but generally the time ranges between 7 to 120 hours post-surgery. ${ }^{12}$ In order to avoid visceral injury, infection and abdominal compartment syndrome, packs are generally removed between 24 to 48 hours after packing, particularly as this time is usually sufficient to apply intensive resuscitation and control bleeding. Nicol et al suggested that removal after 48 hours results in less recurrence of hemorrhage. ${ }^{13}$ Caruso et al found that the risk of bleeding increases when packs are removed before 36 hours. $^{14}$ Abdominal packing maintained beyond 72 hours in 35 women with abdominal trauma showed that removal of pack in $<72$ hours was associated with a statistically significant lower rate of abscess and mortality than removal beyond 72 hours. ${ }^{13,15}$ In the present case sponge holders were used to compress the ongoing bleeding from the cervical stump. Previous case reports suggest abdominal packing as a means of attaining hemostasis but focused compression by sponge holders has not been mentioned in the literature. Such compression provides better and effective compression and the packs are not dislodged and it becomes comparatively easy to remove them on exploration. There was no pressure necrosis and hemostasis was achieved after 48 hours of removal these packs in our case.

\section{Conclusion}

Abdominal and umbrella pack has been used as a means to attain post-hysterectomy hemostasis but focused abdominal compression via sponge holders can also be used to achieve hemostasis as was achieved in this case report but further more studies are needed in this regard to recommend them as a means of focused abdominal compression. 


\section{Acknowledgements}

Dr Neerja Goel, my mentor and guide who is always an inspiration, encouragement and support.

Conflict of Interest: There is no financial interest or any conflict of interest.

Abbreviations: MRI: Magnetic resonance imaging, USG: ultrasonography, PRBC: packed red blood cell, FFP: Fresh frozen plasma, LSCS: Lower segment caesarean section

\section{References}

1. Hughes C, editor. Obstetric -gynecologic terminology: with section on neonatology and glossary on congenital anomalies Philadelphia(PA):F.A Davis;1972.

2. Chou M, Ho E, Lee Y. Prenatal diagnosis of placenta accreta by transabdominal color Doppler ultrasound Ultrasound Obstet Gynecol.2000;15(1):28-35.

3. Post partum heamorrhage. ACOG Bulletin no 76.American college of Obstetrican and Gynecologists. Obstet Gynecol 2006;108:1039-47.

4. Silver R, Landon M, Rouse D, Leveno K, Spong C, Thom E et al. Maternal morbidity associated with multiple repeat cesarean deliveries. Obstet Gynecol. 2006;107(6):1226-32.

5. Warshak C, Eskander, Hull A, Scuoscia A, Mattrey R et al. Accuracy of ultrasonography and magnetic resonance imaging in the diagnosis of placenta accreta. Obstet Gynecol.2006;108:573-81.

6. Comstock C, Love J, Bronstein R, Lee W, Vettrainol et al. Sonographic detection of placenta accreta in the second and third trimesters of pregnancy. Am J Obstet Gynecol 2004;190(4):1135-40.

7. Palacios $\mathbf{J}$ and Bruno $\mathrm{C}$. Magnetic resonance imaging in 300 cases of placenta accrete: a surgical correlation of new findings. Acta Obstet Gynecol Scand. 2005;84:71624.

8. GhourabS. Al-NuaimL, Al-Jabari A, Al Meshari A, Mustafa MS, Abotalib Z, et al. Abdomino-pelvic packing to control severe haemorrhage following caesarean hysterectomy. J Obstet Gynaecol 1.1999;19(2):155-8.

9. Didy G, Scott J, Saffer C, Belfort M et al. An effective pressure pack for severe pelvichaemorrhage secondary to posterior uterine rupture. Obstet Gynecol.2006;100(5):1222-6.

10. Touhami O, Marzaik S, Kehela M, Bennasr L, Fezai A et al. Efficacy and safety of pelvic pack after emergency peripartum hysterectomy (EPP) in post partum haemorrhage (PPH). Eur J Obst Gynecol 2016;202;32-5.

11. Kendrick J, Leath C, Melton S, Straughn M. Use of a fascial prosthesis for management of abdominal compartment syndrome se condary to obstetric hemorrhage. Obstet Gynecol.2006;107(2):493-6.

12. Harp K and Locicero R. Abdominal packing for surgically uncontrollable hemorrhage. Ann Surg. 1992;215(5):467-75.

13. NicolAJ, Hommes M, Primrose R, Navsaria P, KrigeJ. Packing for control of hemorrhage in major liver trauma. World J Surg.2007;31(3):569-74.

14. Caruso D, Battistella F, Owings T, LeeL, Samaco C. Perihepatic packing of major liver injuries: complications and mortality. ArchSurg.1999;134(9):958-62.

15. Abikhaled G, Granchi T, Wall M, Hirshberg et al. Prolonged abdominal packing for trauma is associated with increased morbidity and mortality. Am Surg. 1997;3(2):1109-12. 